\title{
Specific anion effects on adsorption and packing of octadecylamine hydrochloride molecules at the air/water interface
}

\author{
Enze Li ${ }^{\mathrm{a}, \mathrm{b}}$, Xuming Wang ${ }^{\mathrm{c}}$, Zhiping Du ${ }^{\mathrm{a}, \mathrm{b}}$, Jan D. Miller ${ }^{\mathrm{c}, *}$, Fangqin Cheng ${ }^{\mathrm{a}, \mathrm{b}}$ \\ ${ }^{a}$ Institute of Resources and Environment Engineering, State Environmental Protection Key Laboratory of Efficient \\ Utilization Technology of Coal Waste Resources, Shanxi University, No 92 Wucheng Road, Taiyuan, Shanxi \\ 030006, China \\ ${ }^{\mathrm{b}}$ Shanxi Collaborative Innovation Center of High Value-added Utilization of Coal-related Wastes, No 92 Wucheng \\ Road, Taiyuan, Shanxi 030006, China \\ ${ }^{\mathrm{c}}$ Department of Metallurgical Engineering, College of Mines and Earth Sciences, University of Utah, $135 \mathrm{~S} 1460 \mathrm{E}$ \\ 412 WBB, Salt Lake City, UT 84112, USA
}

\section{Corresponding author:}

Jan D. Miller

Ivor D. Thomas Distinguished Professor, Department of Metallurgical Engineering, College of Mines and Earth Sciences, University of Utah, 135 S 1460 E, 412 WBB, Salt Lake City, UT 84112, USA; Phone: +1 801581 5160; Fax: +1 801581 4937; Email: jan.miller@utah.edu

\section{Other authors:}

Enze Li, Email: tustlienze@126.com

Xuming Wang, Email: x.wang@utah.edu

Zhiping Du, Email: duzhiping@sxu.edu.cn

Fangqin Cheng, Email: cfangqin@sxu.edu.cn

(C) 2017. This manuscript version is made available under the Elsevier user license http://www.elsevier.com/open-access/userlicense/1.0/ 


\begin{abstract}
The spread of collector molecules on bubble surfaces in salt solution is a fundamental phenomenon in the froth flotation of soluble salts, such as $\mathrm{KCl}$, and consideration of ionic effects on adsorption behavior and packing of collector molecules at the air/solution interface is appropriate for study. In this work, specific anion effects on the adsorption and packing of one common collector (octadecylamine hydrochloride, ODA) at the air/water interface were investigated by employing a combination of surface pressure measurements, sum frequency vibrational spectroscopy (SFVS) and molecular dynamics simulations (MDS). The results from experiments and MDS showed that the addition of external anions disturb the arrangement of ODA molecules due to the penetration of these anions from the water subphase into the ODA monolayer at the air/water interface. During monolayer compression, the penetrated anions might be driven back to the water subphase by electrostatic forces and the added compression force. These anions could screen the charge of $-\mathrm{NH}_{3}$ groups resulting in a reduction of the minimum area of the ODA molecule $\left(A_{\min }\right)$ for the solid phase state of the monolayer. With the exception of the sulfate anion, the added anions increase hydrogen bonding of interfacial water molecules at the air/water interface. These results allow for a better understanding of the anionic effect on adsorption and packing of ODA molecules at the air/water interface, and might therefore provide new insights into froth flotation of soluble salts and should help to improve soluble salt flotation technology.
\end{abstract}

Keywords: specific anion effects; adsorption at the air/water interface; packing; octadecylamine hydrochloride; monolayer 


\section{Introduction}

Froth flotation continues to be a major industrial process for the production of potash from low grade and complex resources [1]. During processing of certain potash resources, a mixture of sylvite $(\mathrm{KCl})$ and halite $(\mathrm{NaCl})$ is first crystallized by evaporation, and then the $\mathrm{KCl}$ is separated from residual $\mathrm{NaCl}$ by froth flotation [2]. For this industrial process, octadecylamine hydrochloride (ODA) is used as an effective collector [3] and, as all collector molecules, is composed of one hydrophilic head group and one hydrophobic chain. In general, during the process of froth flotation, he hydrophilic head group of collector molecules can adsorb at a particular particle surface while the hydrophobic chains are accommodated by the bubbles [4]. Then these particles are separated with the bubbles due to their bouyancy.

In the process of froth flotation, the bubble-particle interaction must be strong enough to ensure mineral particles can be picked up [5]. Usually, the added collector adsorbs at the mineral surface spontaneously and renders the mineral surface more hydrophobic [6]. However, for the flotation of soluble salts, the solubility of most collectors is very low in saturated brines. Thus, the diffusion of collector molecules/ions and subsequent adsorption at the salt surface become much more difficult [7]. Burdukova and colleagues [8] have reported that long chain primary amines in the saturated salt solutions form colloidal particles. The majority of these colloidal collector particles prefer to directly adsorb at the bubble surface rather than at the KCl crystal surface $[9,10]$. The bubbles with collector are more active and have a strong positive influence on the probability of bubble attachment at the $\mathrm{KCl}$ surface. The attachment between bubbles and $\mathrm{KCl}$ particles allows the collector to be transferred from the bubble surface to the $\mathrm{KCl}$ surface and then the adsorption of the collector at the $\mathrm{KCl}$ surface obviously can be enhanced [11]. The adsorption of collector at the bubble (air/water) surface becomes a prerequisite for the accomplishment of $\mathrm{KCl}$ flotation. Laskowski [12] proposed that the long chain amine deposited at the gas/liquid interface starts rearranging and spreading into a molecular film and then the bubble-collector-KCl crystal structure will form after attachment between these active bubbles and the $\mathrm{KCl}$ crystal surfaces. Therefore, the adsorption, spreading and packing behavior of ODA at the bubble surface will strongly affect the attachment between bubbles and $\mathrm{KCl}$ crystal surfaces, which in turn affects the recovery of $\mathrm{KCl}$. 
It is well known that, in China, most of the $\mathrm{KCl}$ is produced by froth flotation from salt lakes. Actually, the brine for $\mathrm{KCl}$ flotation consists of not only $\mathrm{KCl}$ and $\mathrm{NaCl}$ but also lots of other ions [13]. Anions with larger size and more polarizability, have a propensity for the air/water interface [14], which probably generates an external electric field and reorders the microstructure of water molecules at the interface. These various anions will interact with the ODA molecules introduced at air/water interface, and influence the molecular spreading or packing density of ODA. Although numerous previous works reported the specific ion effects on the behavior of charged monolayers at the air/water interface [15-17], the transfer process of anions and the way these anions act on packing of the ODA monolayer have not been fully understood and require further investigation. Along this line, specific anionic effects on the adsorption and packing of the ODA monolayer at the air/water interface were investigated by using surface pressure measurements, sum frequency vibrational spectroscopy (SFVS) spectra and molecular dynamics simulations (MDS). Both monatomic anions ( $\mathrm{Cl}^{-}, \mathrm{Br}^{-}$and $\left.\mathrm{I}^{-}\right)$and multiatomic anions

$\left(\mathrm{NO}_{3}{ }^{-}, \mathrm{SCN}^{-}\right.$and $\left.\mathrm{SO}_{4}{ }^{2-}\right)$ were considered for comparison. This work may shed light on the relationship between the flotation phenomena and packing of the ODA monolayer at the bubble surface (air/water interface) in the presence of various anions and provide complementary information to explain the flotation of $\mathrm{KCl}$ with ODA.

\section{Experimental section}

\subsection{Materials}

Octadecylamine hydrochloride (ODA) was purchased from Sigma-Aldrich. NaCl, NaI and NaSCN were obtained from Fisher Chemical. $\mathrm{NaBr}$ was purchased from Alfa Aesar, $\mathrm{Na}_{2} \mathrm{SO}_{4}$ was from Mallinckrodt, and $\mathrm{NaNO}_{3}$ was obtained from Amresco. All chemicals used in the experiments were of Anal-R grade and were used without further purification. Milli-Q water was used in the process of all experiments.

\subsection{Surface pressure measurements}

Surface pressure measurements were carried out using a Langmuir Trough (MicroTrough XS, Kibron Inc.) with a volume of $120 \mathrm{~mL}$ and total surface area of $12308 \mathrm{~mm}^{2}$. The ODA sample was prepared by dissolving a powder sample in pure chloroform solvent with a concentration of $0.5 \mathrm{mg} / \mathrm{mL}$. All the salts were dissolved in distilled water with a concentration of $1 \mathrm{mM}$ except 
$\mathrm{Na}_{2} \mathrm{SO}_{4}(0.5 \mathrm{mM})$. Before each measurement, the trough, two barriers and the DyneProbe were washed using acetone, ethyl alcohol and distilled water in turn for several times. Then the trough and barriers were dried with ultra high purity $\mathrm{N}_{2}$ gas and the DyneProbe was further cleaned by flaming. For each measurement the trough was filled with salt solution first. The monolayer was prepared by touching drops of ODA solution $(40 \mu \mathrm{L})$ to the salt solution surface by using a micro-syringe. After waiting for at least $10 \mathrm{~min}$ to ensure complete evaporation of the chloroform solvent, the monolayer was compressed at a rate of $4.0 \AA^{2} / \mathrm{chain} / \mathrm{min}$ and the surface pressure was measured by the sensor. Each measurement was conducted at least three times, in order to ensure the reproducibility of the results.

\subsection{Sum frequency vibrational spectroscopy measurements}

Sum frequency vibrational spectroscopy (SFVS) measurements at the air/water interface were conducted using a sum-frequency generation spectrometer (EKSPLA, Ltd). The laser system has been described in detail in our previous work [18]. The incident angles of the IR beams and visible beams at the air/water interface were 55 and $60^{\circ}$, respectively, and the SFG signal was collected in the reflection mode at an angle of $60^{\circ}$. Energy of the IR and visible beams were $500 \mu \mathrm{J}$ and $160 \mu \mathrm{J}$, respectively. The spectra were collected in SSP polarization combination of the three beams (S: sum-frequency, S: visible, P: infrared), and then were normalized to the IR and visible energies. Data were collected at $5 \mathrm{~cm}^{-1}$ increments and each point is the average of 40 laser shots.

\subsection{Molecular dynamics simulation}

In order to obtain more information on the interaction between added anions and the ODA monolayer at the air/water interface, molecular dynamics simulations were conducted systematically. For each system, the same ODA monolayer was placed at the interface of the air/salt solution. The packing of ODA molecules and the initial configuration are shown in Fig. 1. Each salt solution was composed of 1000 water molecules, $40 \mathrm{Na}^{+}$atoms and 40 anions except

$\mathrm{SO}_{4}{ }^{2-}$ (the concentration of each anion solution was about $2 \mathrm{~mol} / \mathrm{L}$ ). For the $\mathrm{Na}_{2} \mathrm{SO}_{4}$ solution, the number of $\mathrm{SO}_{4}{ }^{2-}$ ions was 20 (the concentration of $\mathrm{SO}_{4}{ }^{2-}$ ions was about $1 \mathrm{~mol} / \mathrm{L}$ ). The simulation cell was orthorhombic with the parameters $a=b=33.2 \AA, c=120 \AA, \alpha=\beta=\gamma=90 \circ$, and periodic boundary conditions. 


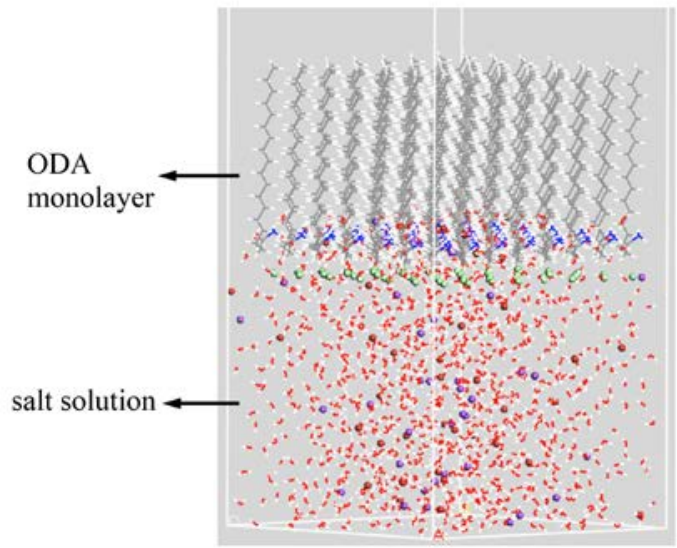

(a)

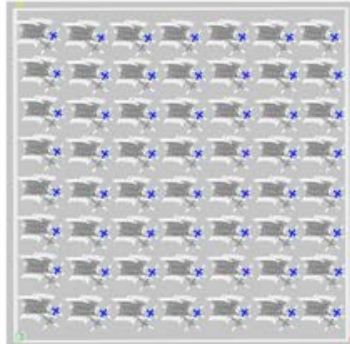

(b)

Fig. 1. (a) The initial configuration of each system (ODA monolayer placed on $\mathrm{NaBr}$ solution, for example), and (b) the packing pattern of ODA molecules in the monolayer (top view, other atoms are omitted for clarity).

The energy of each system was minimized first, and then the minimized system was preequilibrated at ambient conditions in the NVT ( $\mathrm{T}=298 \mathrm{~K}$ ) ensemble for 1 ns with the time step of 1.0 fs. Finally, another $1 \mathrm{~ns}$ simulation with 100 fs intervals was performed, from which the last 200 ps was used to obtain MD trajectory data for further analysis. A PCFF force field [19-23] was used for all simulations and the SPC/E model was used to describe the water molecule [24]. The charge of each atom in the ODA molecule is provided in Fig. 2 and other atomic charges for each element in the salt solutions calculated by the DMol3 package are listed in Table 1.

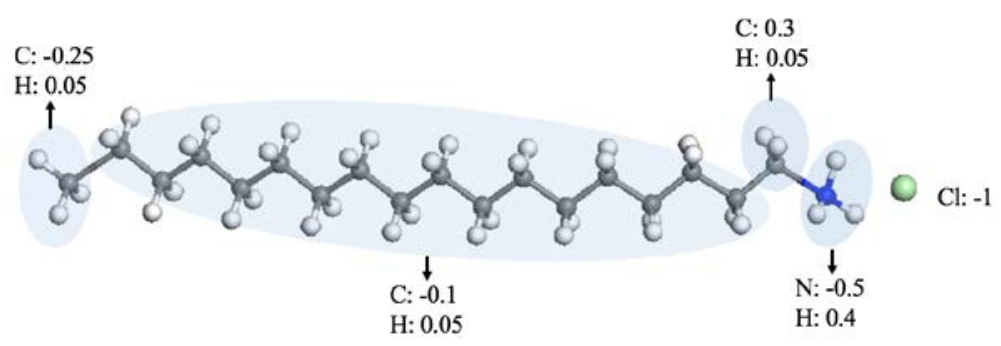

Fig. 2. The charge of each atom in the ODA molecule [25, 26]. 
Table 1. Charge of each element in the salt solution.

\begin{tabular}{ll}
\hline Fragment & Charge of each element \\
\hline $\mathrm{H}_{2} \mathrm{O}$ & $\mathrm{H}: 0.4238 \quad \mathrm{O}:-0.8476$ \\
$\mathrm{Cl}$ & $\mathrm{Cl}:-1$ \\
$\mathrm{Br}$ & $\mathrm{Br}:-1$ \\
$\mathrm{I}$ & $\mathrm{I}:-1$ \\
$\mathrm{NO}_{3}$ & $\mathrm{~N}: 0.779$ O: -0.593 \\
$\mathrm{SCN}$ & $\mathrm{S}:-0.513$ C: $0.097 \mathrm{~N}:-0.585$ \\
$\mathrm{SO}_{4}$ & $\mathrm{~S}: 1.082$ O: -0.771 \\
$\mathrm{Na}$ & $\mathrm{Na}: 1$ \\
\hline
\end{tabular}

\section{Results and discussion}

The results from surface pressure measurements, sum frequency vibrational spectroscopy, and molecular dynamics simulation for ODA at the air/water interface are reported and discussed.

\section{1 $\pi$-A isotherms of ODA monolayer}

Surface pressure $(\pi)$ is the difference in surface tension measured between a clean sub-phase and a monolayer-covered sub-phase. The typical surface pressure-area $(\pi$-A) isotherm can provide the packing density of a monolayer of molecules in the process of compression. Fig. 3 shows the $\pi$-A isotherm for the ODA monolayer on DI water. It can be seen that, under compression, the ODA monolayer starts from a two-dimensional gas phase, and then dramatically transfers to a fully organized solid phase. For the gas phase, most of the ODA molecules are spread on the air/water interface and each molecule occupies a relatively large area which results in no surface pressure. The surface pressure appears when the area per molecule decreases to $11.9 \AA^{2}$ which corresponds to the phase transition point. In the solid phase, the ODA molecules are completely organized and the surface pressure increases dramatically until reaching a maximum value. The minimum area per ODA molecule is $10.4 \AA^{2}$ and after that point the collapse will happen, resulting in the monolayer packing no longer being controlled. For convenience, we define the point at which the surface pressure appears as $A_{0}$, which is also the phase transition point from the gas phase to the liquid phase or directly to the solid phase. In addition, we also define the point at which the surface pressure reaches a maximum value as $A_{\text {min }}$, which corresponds to the minimum area per molecule before collapse (Fig. 3). 


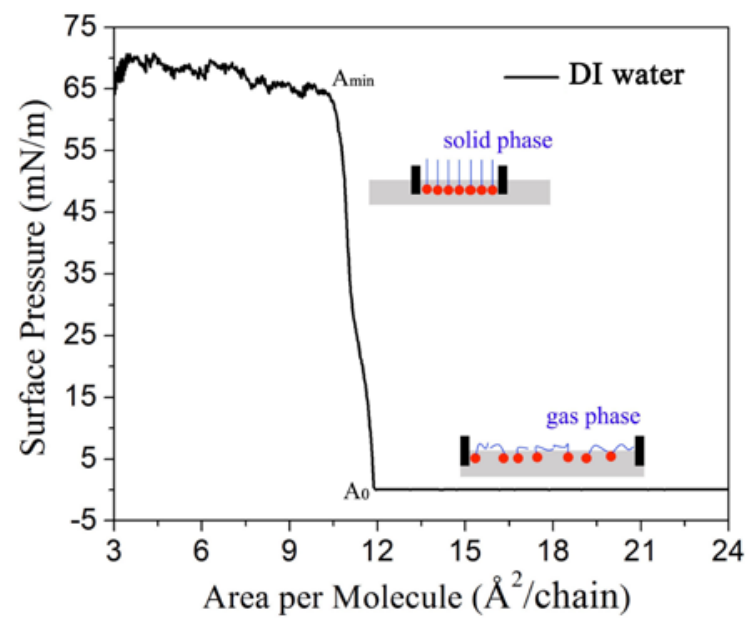

Fig. 3. $\pi$-A isotherm for the ODA monolayer on DI water.
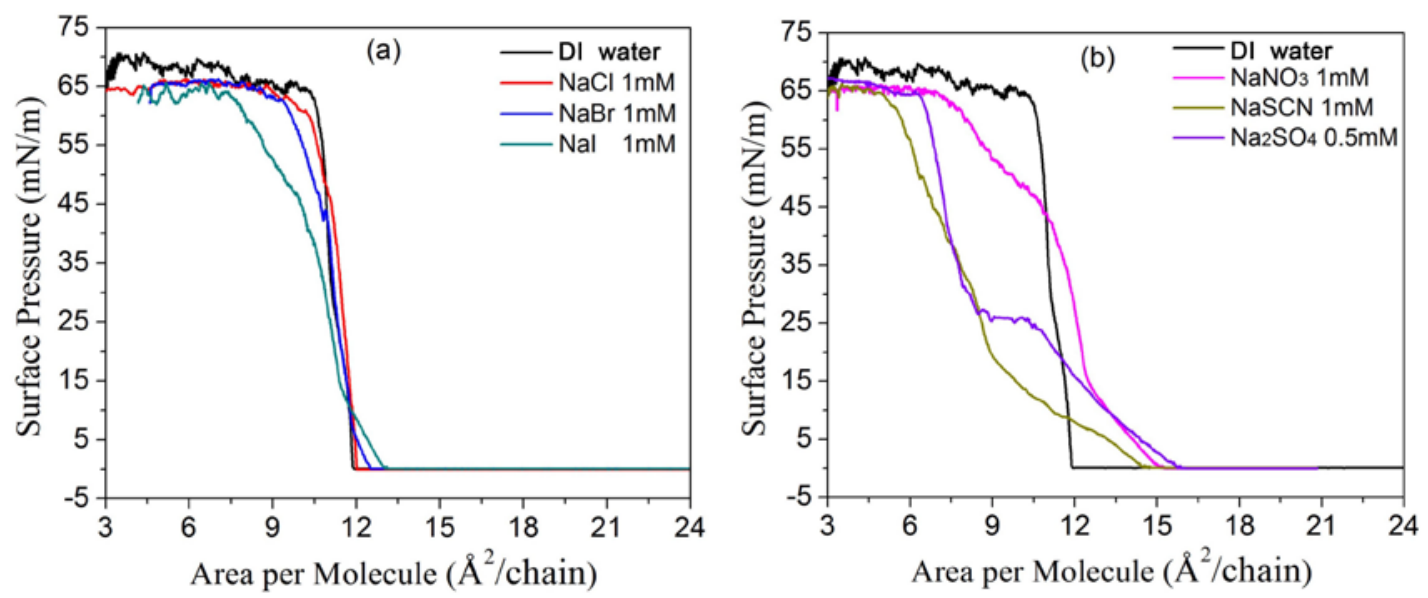

Fig. 4. $\pi$-A isotherms for ODA monolayers with different salt solutions.

A series of $\pi$-A isotherms of ODA monolayers for different salt solutions are presented in Fig. 4. In order to study the effect of anions, $\mathrm{Na}^{+}$is selected as the common cation for all salt solutions with the same concentration. From Fig. 4, the $\pi$-A isotherms exhibit the transformation from gas phase through liquid phase to the solid phase. It can be seen that the $\pi$-A isotherms with $\mathrm{Cl}^{-}, \mathrm{Br}^{-}$and $\mathrm{I}^{-}$are similar to that of the ODA monolayer on DI water (Fig. 4(a)). The transition points from gas phase to liquid phase, $\mathrm{A}_{0}$, for $\mathrm{Cl}^{-}, \mathrm{Br}^{-}$and $\mathrm{I}^{-}$are 12.0, 12.5 and $12.9 \AA^{2}$, respectively, which are slightly larger than that for the DI water system (Table 2). It is noted that the $A_{0}$ increases with the increase of hydrodynamic radii of anions [27]. This may be ascribed to the adsorption of anions at the air/solution interface from the sub-phase before the spreading of the ODA monolayer $[14,28,29]$. Therefore, a few anions at the air/solution interface also 
occupy a certain area resulting in a slightly larger $\mathrm{A}_{0}$ for the ODA monolayer on salt solutions than the $\mathrm{A}_{0}$ on DI water.

In the process of compression, the distance between anions adsorbed at the air/solution interface and head groups of ODA molecules decreases. The anions are probably redistributed in the surrounding area of the ODA head groups due to electrostatic forces and the added compression force. The anions which have interacted with the head groups can screen the electrostatic repulsion between head groups, resulting in much larger packing density of ODA molecules. Therefore, the minimum area for the ODA molecule before collapsing $\left(A_{\min }\right)$ becomes smaller than that for the ODA monolayer on DI water (Table 2). The transfer process of anions from the air/solution interface into the bulk solution is depicted in Fig. 5. In addition, $\mathrm{I}^{-}$ influences the $\mathrm{A}_{\min }$ most among the three halide anions. This is expected to be due to the larger size and polarizability of $\mathrm{I}^{-}$ions [30-32].

Table 2. The $A_{0}$ and $A_{\min }$ of each system.

\begin{tabular}{ccc}
\hline Systems & $\mathrm{A}_{0}\left(\AA^{2}\right)$ & $\mathrm{A}_{\min }\left(\AA^{2}\right)$ \\
\hline $\mathrm{H}_{2} \mathrm{O}$ & 11.9 & 10.4 \\
$\mathrm{NaCl}$ & 12.0 & 9.8 \\
$\mathrm{NaBr}$ & 12.5 & 8.9 \\
$\mathrm{NaI}$ & 12.9 & 7.3 \\
$\mathrm{NaNO}_{3}$ & 15.1 & 6.9 \\
$\mathrm{NaSCN}$ & 14.5 & 4.8 \\
$\mathrm{Na}_{2} \mathrm{SO}_{4}$ & 15.8 & 6.2 \\
\hline
\end{tabular}

Fig. 4(b) shows the $\pi$-A isotherms with $\mathrm{NO}_{3}{ }^{-}, \mathrm{SCN}^{-}$and $\mathrm{SO}_{4}{ }^{2-}$ and the results differ significantly from that of the DI water system. These anions are composed of different atoms and are more complex than those containing only one atom. The $A_{0}$ values of these $\pi$-A isotherms become much larger than those observed for the halide anion system, indicating more anions interact with ODA molecules at the air/solution interface. Along with the compression, the transfer of anions from the air/solution interface into the bulk phase becomes slower, especially for $\mathrm{SO}_{4}{ }^{2-}$ ions, and as a result the rate of surface pressure increase of the ODA monolayer becomes slower, which is not like that of the DI water system. The smaller $A_{\text {min }}$ from Fig. 4(b) 
indicates these anions possess stronger affinity with the amine head groups of ODA compared to interaction with halide anions.

From the energy minimization of the ODA molecule using MOPAC, the theoretical area of the $-\mathrm{NH}_{3}{ }^{+}$group $\left(\sim 3.44 \AA^{2}\right)$ was determined. Although the presence of $\mathrm{SO}_{4}{ }^{2-}$ ions can significantly reduce the $A_{\min }$ of the ODA monolayer, the value is still larger than the occupied area of a solo ODA molecule.This may be ascribed to the repulsion and steric hindrance between ODA molecules.

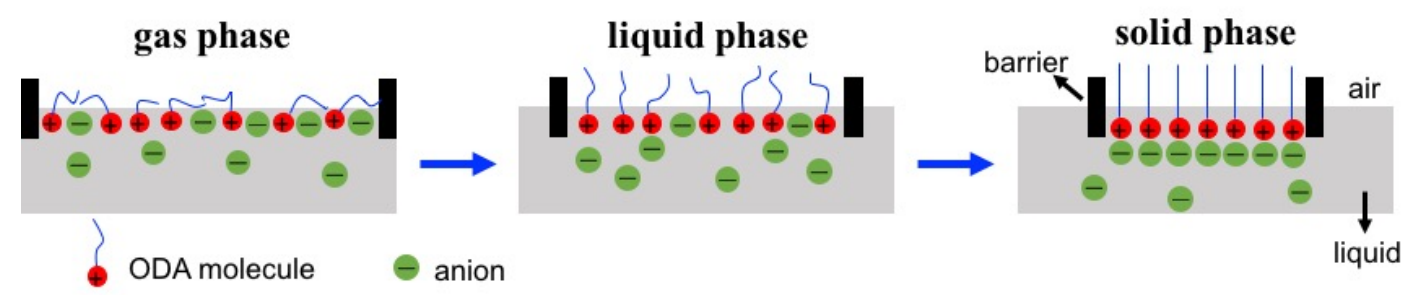

Fig. 5. Transfer of anions during the process of phase transformation for an ODA monolayer on a salt solution.
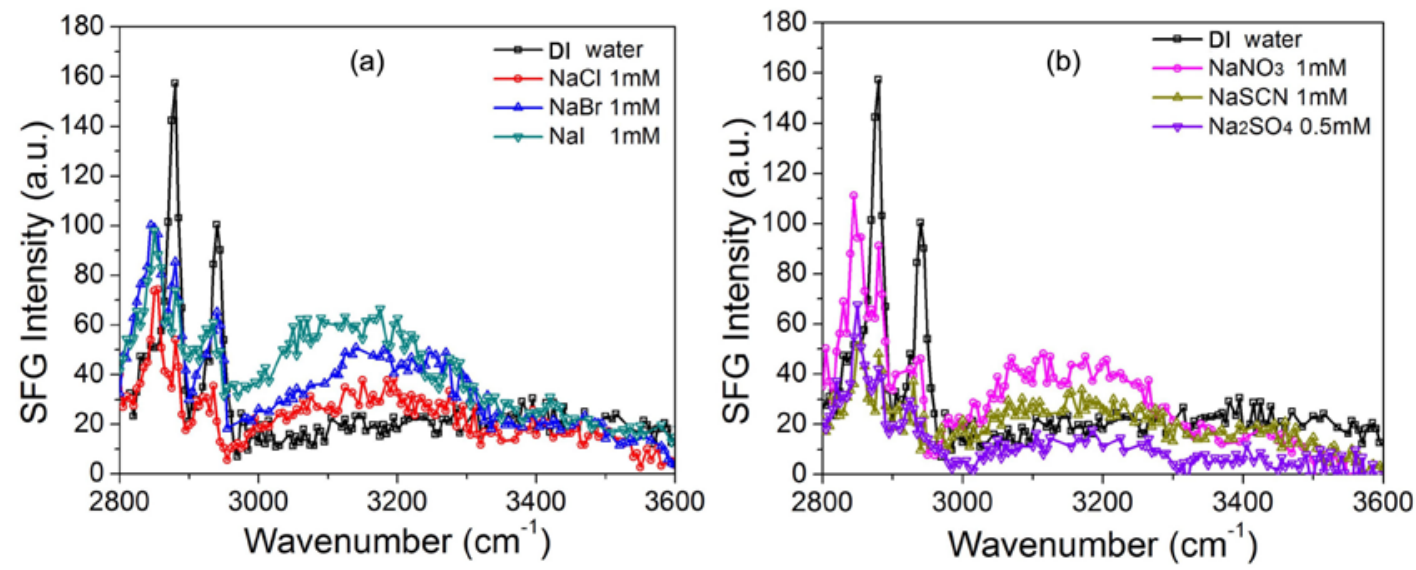

Fig. 6. SFVS spectra under SSP polarization conditions of the air/water interface for ODA solutions prepared in DI water and different salt solutions. The concentration of ODA is $5 \times 10^{-6} \mathrm{~mol} / \mathrm{L}$ for all the solutions tested.

\subsection{Sum frequency vibrational spectroscopy}

In order to obtain more information about the interaction between ODA molecules and different anions, sum frequency vibrational spectroscopy (SFVS) was conducted to monitor the molecular structure of both water and ODA molecules at the air/water interface. Fig. 6 shows the SFVS spectra for the air/water interface of ODA solutions with different anions, together with 
the SFVS spectrum of a solution of ODA in DI water for comparison. According to previous reports [31, 33], the low-frequency range from $2800-3000 \mathrm{~cm}^{-1}$ is mainly assigned to hydrocarbon chains of ODA molecules adsorbed at the air/water interface, and the highfrequency range from $3000-3600 \mathrm{~cm}^{-1}$ corresponds to $\mathrm{OH}$ signals from water molecules at the air/water interface.

Fig. 6 shows that the spectrum for the DI water system has two dominated peaks which are assigned to the $-\mathrm{CH}_{3}$ symmetric stretch $\left(2880 \mathrm{~cm}^{-1}\right)$ and the $-\mathrm{CH}_{3}$ Fermi resonance $\left(2940 \mathrm{~cm}^{-1}\right)$, also a little peak at $2850 \mathrm{~cm}^{-1}$ which is attributed to the $-\mathrm{CH}_{2}$ - symmetric stretch. Another weak broad peak at $3200 \mathrm{~cm}^{-1}$ is assigned to the coupled $\mathrm{OH}$ symmetric stretch from tetrahedrally coordinated water molecules at the interface [33, 34]. These results indicate that the ODA molecules adsorbed at the air/water interface are well-packed and the SFVS spectrum is solely resonances from the chain terminating methyl groups [35], as shown in Fig. 7(a). However, compared with the spectrum from the DI water system, the addition of anions $\left(\mathrm{Cl}^{-}, \mathrm{Br}^{-}, \mathrm{I}\right)$ to the bulk phase decreases the signal from $-\mathrm{CH}_{3}$ and increases the signal from $-\mathrm{CH}_{2}$ - (Fig. 6(a)). It is evident that some gauche defects appear and part of the alkyl - $\mathrm{CH}_{2}$ - groups are exposed to air. This phenomenon may be ascribed to the penetration of anions in the monolayer of ODA molecules resulting in the disorder of interfacial ODA molecules (Fig. 7(b)). In addition, the presence of anions also enhances the signal from the $\mathrm{OH}$ band $\left(3200 \mathrm{~cm}^{-1}\right)$ for all three salt solutions. The increase of the $\mathrm{OH}$ band is modest for $\mathrm{Cl}^{-}$, quite appreciable for $\mathrm{Br}^{-}$and most significant for $\mathrm{I}^{-}$, indicating a greater number of $\mathrm{I}^{-}$ions adsorb at the air/water interface and generate a larger external electric field. The water dipoles near the interface can be aligned by the external electric field, and account for an increase in the signal from the $\mathrm{OH}$ vibration.

(a)

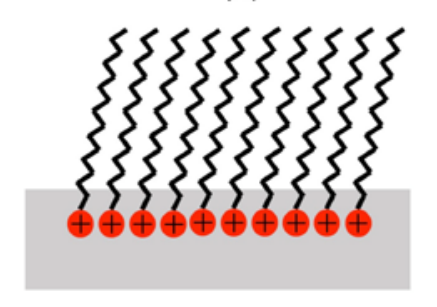

(b)

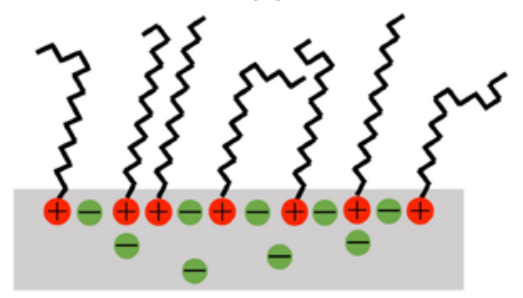

Fig. 7. Arrangement of ODA molecules at the air/water interface, with the bulk solution being (a) DI water; (b) salt solution. 
From Fig. 6(b), the addition of $\mathrm{NO}_{3}{ }^{-}, \mathrm{SCN}^{-}$and $\mathrm{SO}_{4}{ }^{2-}$ can also disturb the arrangement of ODA molecules adsorbed at the air/water interface, resulting in an increase in the SFG signal for $-\mathrm{CH}_{2}$ - and a decrease for $-\mathrm{CH}_{3}$. These results also indicate the penetration of the anions in the ODA monolayer at the air/water interface. However, the three anions show different effects on the signal from the $\mathrm{OH}$ band. The $\mathrm{NO}_{3}{ }^{-}$can remarkably enhance the intensity of the $\mathrm{OH}$ band, and $\mathrm{SCN}^{-}$has no significant influence, while the signal of the $\mathrm{OH}$ band shows a slight decrease with the addition of $\mathrm{SO}_{4}{ }^{2-}$. Compared with other anions, $\mathrm{SO}_{4}{ }^{2-}$ ions have a high tendency to transfer to the positively charged air/water interface because of their divalent character [36]. These interfacial $\mathrm{SO}_{4}{ }^{2-}$ ions can weaken the $\mathrm{H}_{2} \mathrm{O}-\mathrm{H}_{2} \mathrm{O}$ interactions due to their more disruptive nature on the water structure [36, 37]. Because the $\mathrm{SO}_{4}{ }^{2-}$ ion has a larger solvation shell and solvation enthalpy than other anions $[27,38]$, the presence of $\mathrm{SO}_{4}{ }^{2-}$ will disturb the structure of tetrahedrally coordinated water molecules, resulting in a weaker intensity of the $\mathrm{OH}$ band.

\subsection{Molecular dynamics simulation}

Molecular dynamics simulations (MDS) provide an effective method for the generation of complementary information at the atomic level, which is difficult to obtain experimentally [3942]. The behavior of an ODA monolayer at the air/salt solution interface and the effect of anions have been further investigated through MDS. Fig. 8 shows the density profiles of atoms/molecules along the z-axis for $\mathrm{NaI}$ and $\mathrm{Na}_{2} \mathrm{SO}_{4}$ systems. From Fig. 8, it can be seen that the distribution of $\mathrm{Cl}$ atoms originating from ODA molecules is almost similar to that of $-\mathrm{NH}_{3}$ for both systems, suggesting most of these $\mathrm{Cl}$ atoms still surround and interact with the $-\mathrm{NH}_{3}$ groups. However, for both the $\mathrm{I}^{-}$and $\mathrm{SO}_{4}{ }^{2-}$ anions originating from the $\mathrm{NaI}$ and $\mathrm{Na}_{2} \mathrm{SO}_{4}$ solutions,

respectively, the anions appear throughout the considered range of Z-axis and their density curves overlap with those for hydrocarbon alkyl chains of the ODA molecules. These phenomena indicate that both the $\mathrm{I}^{-}$and $\mathrm{SO}_{4}{ }^{2-}$ anions can be divided into three parts on the basis of their disposition in the systems considered. One part is still dispersed in the water, another part will tend to interact with the cationic head groups of ODA through electrostatic forces, and the other part can adsorb at the air/solution interface and penetrate into the ODA monolayer, which may disturb the arrangement of ODA molecules. 

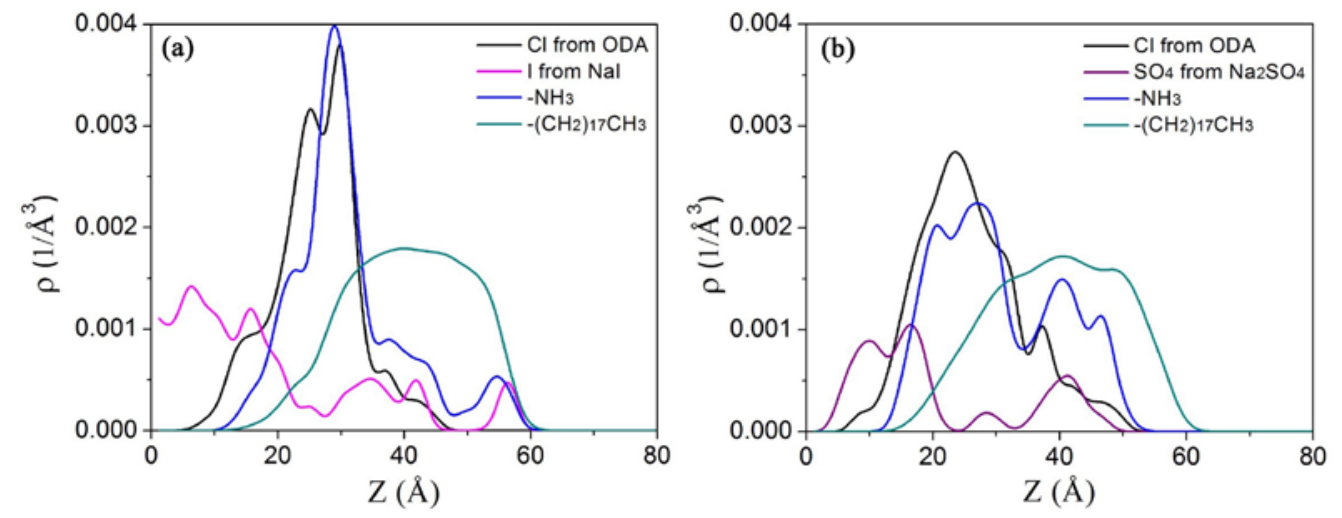

Fig. 8. Density profiles along the $z$-axis for $\mathrm{NaI}$ and $\mathrm{Na}_{2} \mathrm{SO}_{4}$ systems. The bottom of the simulation cell is defined as $z=0$. Black: $\mathrm{Cl}$ of ODA molecule; Blue: $-\mathrm{NH}_{3}$, head group of ODA; Dark cyan: - $\left(\mathrm{CH}_{2}\right)_{17} \mathrm{CH}_{3}$, hydrocarbon alkyl chain of ODA molecule; the other color is assigned to the anions of salt solution for each system.

Fig. 9 shows the final configuration of systems with $\mathrm{I}^{-}$and $\mathrm{SO}_{4}{ }^{2-}$. It is apparent that part of the anions can transfer from the corresponding salt solution to the air/water interface and be inserted into the monolayer, especially for $\mathrm{SO}_{4}{ }^{2-}$, resulting in ODA molecules with much less order. Compared with other anions, a much greater percent of the $\mathrm{SO}_{4}{ }^{2-}$ anions interact with the ODA head groups or penetrate into the ODA monolayer, which is consistent with the results from SFVS measurements.

(a)

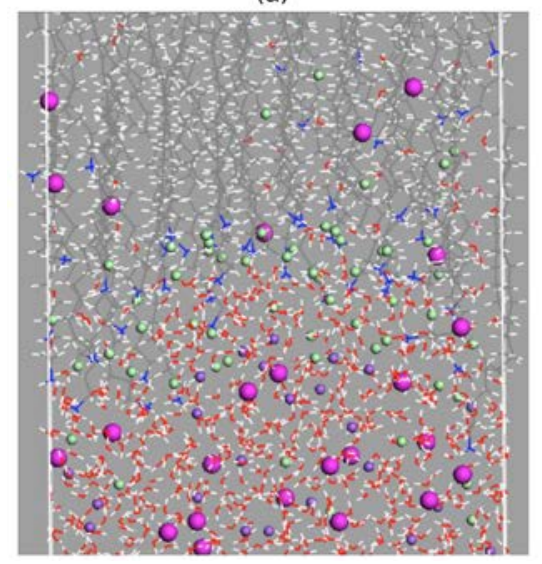

(b)

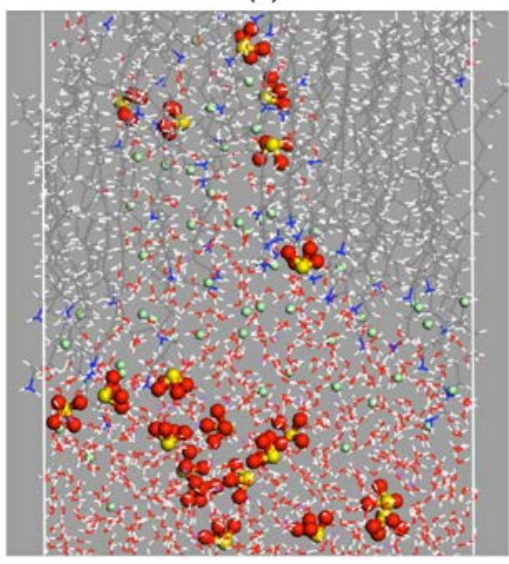

Fig. 9. MDS snapshots of (a) NaI system and (b) $\mathrm{Na}_{2} \mathrm{SO}_{4}$ system after simulation (side view). The atoms' color codes are as follow: magenta, I; yellow, S; red, O; blue, N; green, Cl; violet, Na; white, H; black, C. 

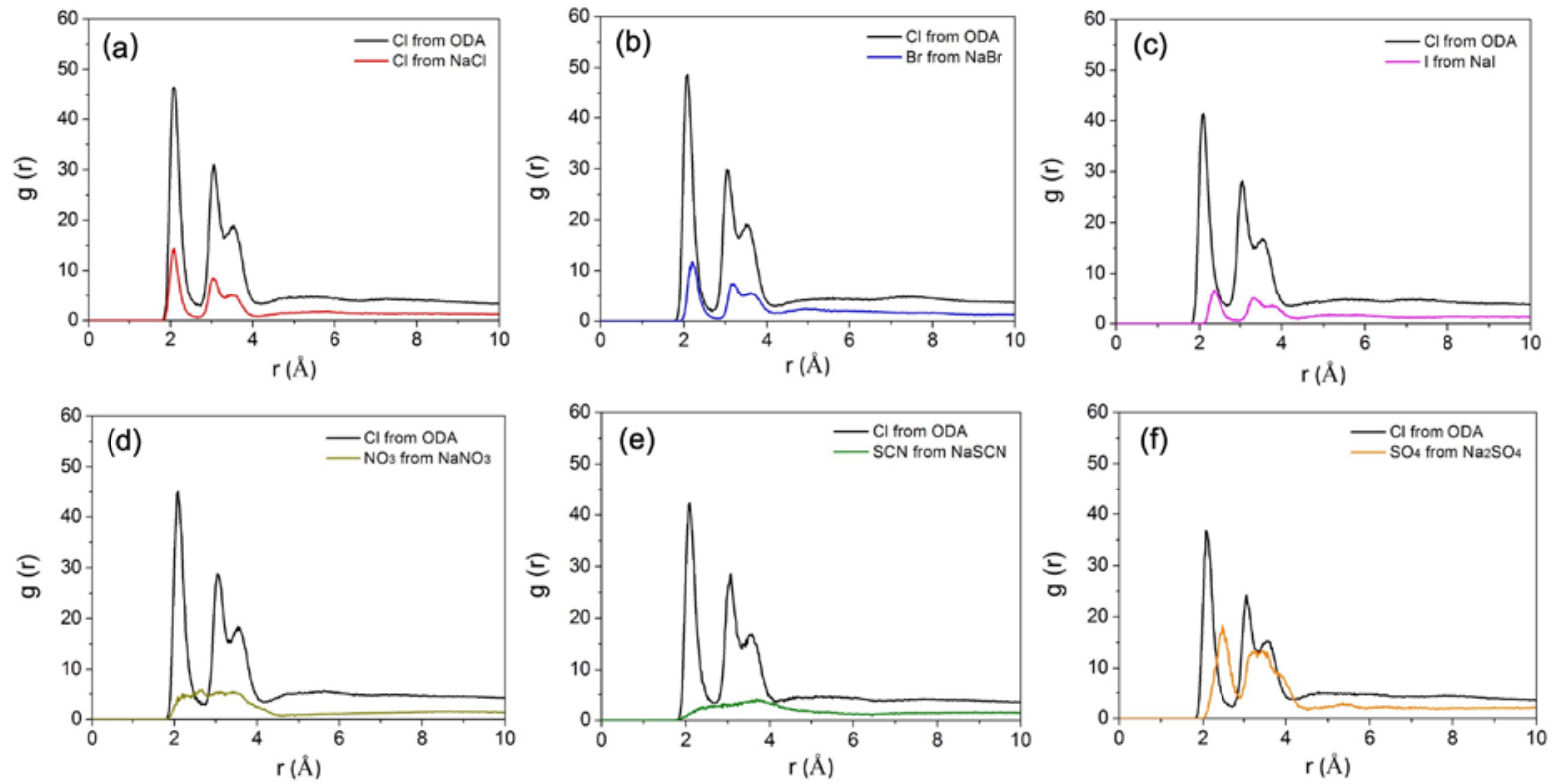

Fig. 10. The radial distribution function of head groups of ODA $\left(-\mathrm{NH}_{3}\right)$ and anions for different systems.

The distribution of anions around the head group $-\mathrm{NH}_{3}$ can be determined by the radial distribution function (RDF), which can help us evaluate the interaction between anions and $-\mathrm{NH}_{3}$. Fig. 10 shows the $\mathrm{RDF}$ of $-\mathrm{NH}_{3}$ and anions for different systems. We observe that all the curves for $\mathrm{Cl}^{-}$ions originated from ODA and anions originated from salt solutions have two or three peaks except $\mathrm{NO}_{3}{ }^{-}$and $\mathrm{SCN}^{-}$. Each peak represents a layer of anions around the $-\mathrm{NH}_{3}$ groups and the radii is the distance between the anion layer and $-\mathrm{NH}_{3}$ groups. Thus, there is no tight anion layer around $-\mathrm{NH}_{3}$ groups formed by $\mathrm{NO}_{3}{ }^{-}$and $\mathrm{SCN}^{-}$. It is noted that the first peak of these RDFs is the most valuable to interpret the interaction between $-\mathrm{NH}_{3}$ and the anions. From Fig. 10, it is clear that the peaks for the $\mathrm{Cl}^{-}$ions originated from ODA are significantly higher than those for anions in the salt solutions, indicating that the $-\mathrm{NH}_{3}$ groups are still inclined to interact with the $\mathrm{Cl}^{-}$ions from the original ODA.

For all the simulation systems, the average cover area of each ODA molecule is $19.68 \AA^{2}$ and the corresponding phase for the ODA monolayer is the two-dimension gas phase on the basis of the results from surface pressure measurements. From Fig. 10(a-c), for the curves of $\mathrm{Cl}^{-}, \mathrm{Br}^{-}$and $\mathrm{I}^{-}$, the radii of the first peak gradually increase as the intensity gradually decreases, although the difference has no significance. This might be because the I' ions surrounding the $-\mathrm{NH}_{3}$ groups prefer to adsorb at the air/water interface and exist within the ODA chains rather than directly interact with $-\mathrm{NH}_{3}$ groups, when the ODA monolayer is described as a gas phase. 
Compared with curves for $\mathrm{NO}_{3}{ }^{-}$and $\mathrm{SCN}^{-}$, the curve of $\mathrm{SO}_{4}{ }^{2-}$ has two distinguishable strong peaks with the first peak located at $2.48 \AA$ (Fig. 10(d-e)). Apparently, the radii and intensity of the first peak for $\mathrm{SO}_{4}{ }^{2-}$ is greater and higher, respectively, than any other of the anions from the salt solutions. The results suggest the insertion of more $\mathrm{SO}_{4}{ }^{2-}$ anions among ODA chains at the air/water interface.

\section{Conclusions}

Our study shows specific effects of various anions on the adsorption and packing of the ODA monolayer at the air/water interface. Surface pressure measurements demonstrate that some of anions can transfer from salt solutions to the air/water interface and penetrate into the ODA monolayer before compression, especially for $\mathrm{I}^{-}$and $\mathrm{SO}_{4}{ }^{2-}$ due to their larger size and higher polarizability. At the final stage of compression, the presence of anions induces smaller $A_{\text {min }}$ before collapsing and the influence by $\mathrm{SO}_{4}{ }^{2-}$ is most significant. This is evidence that the penetrated anions within the ODA monolayer may be driven back to the bulk phase by the electrostatic force and the added compression force, and then screen the charge of $-\mathrm{NH}_{3}$ groups, which results in a reduction of the repulsion between these head groups. The SFVS studies also demonstrate the penetration of anions among ODA chains which will disturb the arrangement of ODA molecules and induce the presence of gauche defects. It is noted that the signal of the $\mathrm{OH}$ band from the water molecules is enhanced with the addition of $\mathrm{Cl}^{-}, \mathrm{Br}^{-}, \mathrm{I}^{-}$and $\mathrm{NO}_{3}{ }^{-}$due to the external electric field. However, $\mathrm{SO}_{4}{ }^{2-}$, with a larger solvation shell, can break the structure of tetrahedrally coordinated water molecules, resulting in weaker $\mathrm{OH}$ band intensity. In addition, the results from MDS complement experimental surface chemistry results and reveal the existence of anions among ODA chains, when the ODA monolayer is in the two-dimensional gas phase state. We also find that the $\mathrm{Cl}^{-}$ions originating from the ODA still mainly surround the $-\mathrm{NH}_{3}$ groups of ODA rather than being dispersed into the aqueous phase.

\section{Acknowledgments}

This work was financially supported by the National Nature Science Foundation of China (Grant No. 21376144) and by the Division of Chemical Sciences, Geosciences, and Biosciences, Office of Basic Energy Sciences of the US Department of Energy through Grant No. DE-FG0393ER14315. 


\section{References}

[1] H. Du, O. Ozdemir, X. Wang, F. Cheng, M. Celik, J. Miller, Flotation chemistry of soluble salt minerals: from ion hydration to colloid adsorption, Miner. Metall. Proc. 31 (2014) 1-20.

[2] X. Wang, J.D. Miller, F. Cheng, H. Cheng, Potash flotation practice for carnallite resources in the Qinghai Province, PRC, Miner. Eng. 66 (2014) 33-39.

[3] X. Diao, H. Zhang, J. Zhang, F. Cheng, Spectrophotometric determination of octadecylamine in brine from KCl flotation, J. North Univ. China (Nat. Sci. Ed.) 3 (2011) 017.

[4] W. Liu, X. Wang, Z. Wang, J.D. Miller, Flotation chemistry features in bastnaesite flotation with potassium lauryl phosphate, Miner. Eng. 85 (2016) 17-22.

[5] M. Gharabaghi, S. Aghazadeh, A review of the role of wetting and spreading phenomena on the flotation practice, Curr. Opin. Colloid In. 19 (2014) 266-282.

[6] F. Li, H. Zhong, G. Zhao, S. Wang, G. Liu, Adsorption of $\alpha$-hydroxyoctyl phosphonic acid to ilmenite/water interface and its application in flotation, Colloid. Surface. A 490 (2016) 67-73.

[7] Z. Wu, X. Wang, H. Liu, H. Zhang, J.D. Miller, Some physicochemical aspects of water-soluble mineral flotation, Adv. Colloid Interfac. 235 (2016) 190-200.

[8] E. Burdukova, J. Laskowski, Effect of insoluble amine on bubble surfaces on particle-bubble attachment in potash flotation, Can. J. Chem. Eng. 87 (2009) 441-447.

[9] J. Leja, J.H. Schulman, Flotation theory: molecular interactions between frothers and collectors at solid-liquid-air interfaces, Trans. Am. Inst. Min., Metall. Petrol. Eng. 199 (1954) 221-228.

[10] C. Smolders, E. Duyvis, Contact angles; wetting and de-wetting of mercury: Part I. A critical examination of surface tension measurement by the sessile drop method, Recl. Trav. Chim. Pays-Bas 80 (1961) 635-649.

[11] E. Li, Y. Zhang, Z. Du, D. Li, F. Cheng, Bubbles facilitate ODA adsorption and improve flotation recovery at low temperature during $\mathrm{KCl}$ flotation, Chem. Eng. Res. Des. 117 (2017) 557-563.

[12] J.S. Laskowski, From amine molecules adsorption to amine precipitate transport by bubbles: A potash ore flotation mechanism, Miner. Eng. 45 (2013) 170-179.

[13] W. Liu, H. Xu, X. Shi, X. Yang, Fractional crystallization for extracting lithium from Cha'erhan tail brine, Hydrometallurgy 167 (2017) 124-128.

[14] P. Jungwirth, D.J. Tobias, Ions at the air/water interface, J. Phys. Chem. B 106 (2002) 6361-6373.

[15] V.L. Shapovalov, Interaction of DPPC monolayer at air-water interface with hydrophobic ions, Thin Solid Films 327-329 (1998) 599-602.

[16] A.M. Brzozowska, M.H.G. Duits, F. Mugele, Stability of stearic acid monolayers on Artificial Sea Water, Colloid. Surface. A 407 (2012) 38-48.

[17] W. Wang, W. Sung, M. Ao, N.A. Anderson, D. Vaknin, D. Kim, Halide ions effects on surface excess of long chain ionic liquids water solutions, J. Phys. Chem. B 117 (2013) 13884-13892.

[18] Z.S. Nickolov, X. Wang, J.D. Miller, Liquid/air interfacial structure of alcohol-octyl hydroxamic acid mixtures: a study by sum-frequency spectroscopy, Spectrochim. Acta A 60 (2004) 2711-2717.

[19] H. Sun, Force field for computation of conformational energies, structures, and vibrational frequencies of aromatic polyesters, J. Comput. Chem. 15 (1994) 752-768. 
[20] H. Sun, S.J. Mumby, J.R. Maple, A.T. Hagler, An ab initio CFF93 all-atom force field for polycarbonates, J. Am. Chem. Soc. 116 (1994) 2978-2987.

[21] H. Sun, S. Mumby, J. Maple, A. Hagler, Ab initio calculations on small molecule analogs of polycarbonates, J. Phys. Chem. 99 (1995) 5873-5882.

[22] S.H. Min, S.K. Kwak, B.S. Kim, Atomistic simulation for coil-to-globule transition of poly (2dimethylaminoethyl methacrylate), Soft matter, 11 (2015) 2423-2433.

[23] Y. Xu, Y.L. Liu, D.D. He, G.S. Liu, Adsorption of cationic collectors and water on muscovite ( $\left(\begin{array}{lll}0 & 1\end{array}\right)$ surface: A molecular dynamics simulation study, Miner. Eng. 53 (2013) 101-107.

[24] W. Liu, X. Wang, H. Xu, J. Miller, Lauryl phosphate adsorption in the flotation of bastnaesite, (Ce, La) $\mathrm{FCO}_{3}$, J. Colloid Interf. Sci. 490 (2016) 825-833.

[25] H. Heinz, H. Koerner, K.L. Anderson, R.A. Vaia, B. Farmer, Force field for mica-type silicates and dynamics of octadecylammonium chains grafted to montmorillonite, Chem. Mater. 17 (2005) 56585669.

[26] Q. Cao, H. Du, J.D. Miller, X. Wang, F. Cheng, Surface chemistry features in the flotation of KCl, Miner. Eng. 23 (2010) 365-373.

[27] Y. Marcus, Thermodynamics of solvation of ions. Part 5.-Gibbs free energy of hydration at 298.15 K, J. Chem. Soc., Faraday Trans. 87 (1991) 2995-2999.

[28] P. Jungwirth, D.J. Tobias, Specific ion effects at the air/water interface, Chem. Rev. 106 (2006) 1259-1281.

[29] B.C. Garrett, Ions at the air/water interface, Science 303 (2004) 1146-1147.

[30] Z. Avazbaeva, W. Sung, J. Lee, M.D. Phan, K. Shin, D. Vaknin, D. Kim, Origin of the instability of octadecylamine Langmuir monolayer at low pH, Langmuir 31 (2015) 13753-13758.

[31] W. Sung, W. Wang, J. Lee, D. Vaknin, D. Kim, Specificity and variation of length scale over which monovalent halide ions neutralize a charged interface, J. Phys. Chem. C 119 (2015) 7130-7137.

[32] S. Nihonyanagi, S. Yamaguchi, T. Tahara, Counterion effect on interfacial water at charged interfaces and its relevance to the hofmeister series, J. Am. Chem. Soc. 136 (2014) 6155-6158.

[33] D. Gragson, B. McCarty, G. Richmond, Ordering of interfacial water molecules at the charged air/water interface observed by vibrational sum frequency generation, J. Am. Chem. Soc. 119 (1997) 6144-6152.

[34] Q. Du, E. Freysz, Y.R. Shen, Vibrational spectra of water molecules at quartz/water interfaces, Phys. Rev. Lett. 72 (1994) 238-241.

[35] A.G. Lambert, P.B. Davies, D.J. Neivandt, Implementing the theory of sum frequency generation vibrational spectroscopy: A tutorial review, Appl. Spectrosc. Rev. 40 (2005) 103-145.

[36] A.J. Hopkins, S. Schrödle, G.L. Richmond, Specific ion effects of salt solutions at the $\mathrm{CaF}_{2} / \mathrm{water}$ interface, Langmuir 26 (2010) 10784-10790.

[37] Y. Marcus, Effect of ions on the structure of water: Structure making and breaking, Chem. Rev. 109 (2009) 1346-1370.

[38] Y. Marcus, The thermodynamics of solvation of ions. Part 2.-The enthalpy of hydration at $298.15 \mathrm{~K}$, J. Chem. Soc., Faraday Trans. 83 (1987) 339-349.

[39] E. Li, Z. Du, S. Yuan, Properties of a water layer on hydrophilic and hydrophobic self-assembled monolayer surfaces: A molecular dynamics study, Sci. China Chem. 56 (2013) 773-781. 
[40] E. Li, Z. Du, G. Wang, F. Cheng, S. Yuan, Aggregation behavior of trisiloxane-tailed surface active ionic liquids in aqueous solution: Coarse-grained molecular dynamics study, J. Disper. Sci. Technol. 35 (2014) 1520-1527.

[41] E. Li, Z. Du, S. Yuan, F. Cheng, Low temperature molecular dynamic simulation of water structure at sylvite crystal surface in saturated solution, Miner. Eng. 83 (2015) 53-58.

[42] G. Lv, F. Gao, G. Liu, S. Yuan, The properties of asphaltene at the oil-water interface: A molecular dynamics simulation, Colloid. Surface. A 515 (2017) 34-40. 